\title{
Is acupuncture an effective postherpetic neuralgia treatment? A systematic review and meta-analysis
}

This article was published in the following Dove Press journal:

Journal of Pain Research

\author{
Wenya Pei' \\ Jingchun Zeng ${ }^{2}$ \\ Liming $\mathrm{Lu}^{3}$ \\ Guohua Lin ${ }^{2}$ \\ Jingwen Ruan'
}

'Department of Acupuncture, the First Affiliated Hospital of Sun Yat-sen University, Guangzhou, Guangdong, People's Republic of China; ${ }^{2}$ Department of Acupuncture, The First Affiliated Hospital of Guangzhou University of Chinese Medicine, Guangzhou, Guangdong, China; ${ }^{3}$ Clinical Medical Center, South China Research Center for Acupuncture and Moxibustion, Medical College of Acu-Moxi and Rehabilitation, Guangzhou University of Chinese Medicine, Guangzhou, Guangdong, China

Correspondence: jingwen Ruan Department of Acupuncture, 3rd Floor of Outpatient Building The First Affiliated

Hospital of Sun Yat-sen University, Zhong Shan Er Lu No.58, Guangzhou,

Guangdong, People's Republic of China

Tel +86 I33। 622 I322

Email ruanjw@।63.com
Background: Postherpetic neuralgia (PHN) refers to pain which remains after the healing of rashes from herpes zoster. Previous literatures have shown that acupuncture has potential benefits for PHN, but evidence remains lacking. Thus, we have performed a systematic review and meta-analysis to identify the effectiveness of acupuncture in the treatment of PHN.

Methods: Six databases were searched for randomized controlled trials (RCTs) to assess the effects of acupuncture on PHN. After selecting the studies, extracting the data, and assessing study quality, meta-analysis was performed on several of the studies with RevMan 5.3. The GRADE (Grading of Recommendations Assessment Development and Evaluation) system was used to assess the overall quality of the evidence.

Results: Acupuncture helps relieve pain intensity (standardized mean difference [SMD]: -1.78 , $95 \%$ confidence interval $[\mathrm{CI}]:-2.36$ to -1.21$)$. For other intervention types, electroacupuncture (SMD: -1.28 , 95\% CI: -2.51 to -0.05 ), fire needle (SMD: $-2.23,95 \%$ CI: -2.62 to -1.84 ), bloodletting and cupping (SMD: $-2.46,95 \%$ CI: -2.95 to -1.97 ) have better effects on pain intensity relief. To date, no study has reported on the onset of pain relief time. The Hamilton Anxiety Scale score (SMD: $-18.94,95 \%$ CI: -37.37 to -0.52 ) was lower for the acupuncture group than for the control group. It was also found that acupuncture can improve quality of life (QOL) (SMD: 3.78, 95\% CI: 2.50 to 5.06). The quality of evidence for acupuncture for PHN pain intensity was moderate according to the GRADE system.

Conclusion: Acupuncture may reduce pain intensity, relieve anxiety and improve quality of life in patients with PHN. Further randomized trials with larger sample sizes and of higher methodological quality are needed to confirm these results.

Keywords: acupuncture, postherpetic neuralgia, systematic review

\section{Introduction}

Postherpetic neuralgia (PHN) is a chronic refractory neuropathic pain syndrome. It is characterized by the invasive nerve distribution area maintaining persistent, severe pain after herpes zoster lesions are gone. ${ }^{1-3} 18-41 \%$ of patients suffering from herpes zoster experience intense pain, the most common chronic complication after the healing of the rash. ${ }^{3-6}$ The pain persists for months or even years, during which physical, emotional and social function may suffer. ${ }^{7,8}$ Increasing age is a risk factor for PHN, and it has been reported that immunosuppression, systemic lupus erythematosus, diabetes and recent trauma can also increase risk. ${ }^{9,10}$

The current pharmacological treatment for PHN is tricyclic antidepressants (amitriptyline, nortriptyline, desipramine and maprotiline), gabapentin, pregabalin, opioids and topical lidocaine patches. ${ }^{1-13}$ However, the anticholinergic side effects 
of tricyclic antidepressants have cardiovascular and renal comorbidities which limit their use. ${ }^{14}$ Occasionally, despite the administration of complex drug combinations, analgesia is still ineffective. ${ }^{15}$

In recent years, clinical and scientific attention to acupuncture has increased. Acupuncture is a reliable and reproducible treatment for acute and chronic pain. ${ }^{16-18}$ Reports have shown the effectiveness of acupuncture in the treatment of chronic pain and that the treatment effects persist for weeks. ${ }^{17,19-21}$ Many reports have confirmed the therapeutic effects of acupuncture on PHN. These have included case reports and pilot studies which have identified acupuncture as a therapy with promising results for relieving pain without significant adverse reactions. ${ }^{22-24}$ As more and more PHN treatments are developed, acupuncture might have an advantage for analgesia and reducing adverse reactions. However, this statement lacks evidence. Thus, we have collected and summarized published evidence on acupuncture for PHN.

\section{Methods}

This review has been registered in the PROSPERO database (PROSPE-RO Register code: CRD42018080847 http://www.crd.york.ac.uk/PROSPER-O/) and has been reported according to the PRISMA Statement. (S1 PRISMA Checklist)

\section{Types of studies}

Any randomized trials providing the highest level of evidence to assess the effects of interventions, whether doubleblind, single-blind or unblinded, and regardless of publication status, language and length of trial were considered for inclusion. We scanned quasi-randomized and other observational studies which were retrieved with searches for randomized clinical trials for reports on harm only. When administering acupuncture, patients are typically aware of the acupoints and methods. This makes blinding difficult. In this study, there were no special requirements for blinding subjects or administrators.

\section{Types of participants}

Participants with PHN, regardless of age, sex or ethnicity, were included in this study. Because its diagnostic criteria are inconsistent, we defined PHN as pain persisting for more than 1 month, or any of the above criteria included into the literature.

\section{Types of interventions}

We included studies that used acupuncture as the experimental group. This included ordinary acupuncture, electro-acupuncture, moxibustion, bloodletting, cupping, needle, fire needle, acupuncture injection, plum blossom needle and ear acupuncture. Studies with no treatment, sham acupuncture, placebo controls or drug therapy were also included. However, studies using treatment in combination with herbal medicine or other nonacupuncture treatments were excluded.

\section{Types of outcome measures}

The primary outcome in this study was pain intensity, as measured by the Visual Analogue Scal (VAS), Numerical Rating Scale (NRS), McGill Pain Questionnaire (MPQ), Verbal Rating Scale (VRS) as well as several other scales for measuring pain. VAS is the most common measurement to assess pain intensity. It is scored on a range of either $0-10$ or $0-100$. NRS ( $0=$ no pain, $10=$ worst pain) and VRS (none/ very mild/mild-moderate/severe/very severe) are similar to VAS in that pain intensity is measured by numbers and descriptions. A higher score indicates greater pain intensity. The MPQ consists of 20 subcategories, in four parts, to measure pain properties and intensity. A higher score means more serious pain.

The secondary outcome included onset of pain relief time, Hamilton Anxiety Scale (HAMA), quality of life (QOL) and reverse effects. The onset of pain relief time is the amount of time before pain relief began. The shorter the time requirement, the more effective the treatment. The HAMA consists of 14 items ranging from 0 to 4 , which assess the severity of patients' anxiety states. A higher score suggests a higher degree of anxiety. In this study, QOL was an assessment method for QOL related to pain, with a range of $0-10$. A higher score meant better QOL.

\section{Search methods for identification of studies}

All literature on acupuncture treatment for PHN was included, with no restriction on language or publishing status (ie, unpublished articles were included). Following the Cochrane Handbook for Systematic Review of Interventions (Version 5.1.0), the search terms used were "postherpetic neuralgia", "acupuncture" and "random".

\section{Electronic searches}

We searched the Cochrane Central Register of Controlled Trials (CENTRAL), MEDLINE, EMBASE (1980-2018), 
Chinese Medical Database (CNKI, 1979.1.1-2018.7.31), Vip Citation Databases (Chongqing Veipu,1989-2018) and Wanfang (1990-2018).

\section{Searching other resources}

We searched the reference lists of the identified trials to identify further relevant trials. We also searched online trial registries such as ClinicalTrials.gov (ClinicalTrials.gov/), European Medicines Agency (EMA) (www.e-ma.europa.eu/ ema/), WHO International Clinical Trials Registry Platform (www.who.int/ictrp) as well as ongoing or unpublished trials.

The search deadline was July 31, 2018, and the search strategy varied based on the character of each database. The search strategy details are shown in the Supporting Information section. (S2 search strategy).

\section{Data collection and analysis} Selection of studies

For this review, one author (Liming Lu) generated the electronic search strategies in the Cochrane Central Register of Controlled Trials (CENTRAL), MEDLINE, EMBASE (1980-2018), Chinese Medical Database (CNKI, 1979.1.12018.7.31), Vip Citation Databases (Chongqing Veipu, 1989-2018) and Wanfang (1990-2018). Liming Lu also downloaded the citations.

Two authors (Wenya Pei and Jingchun Zeng) independently applied the aforementioned inclusion criteria to identify trials with EndNote X6.0. Any disagreements between authors were resolved with arbitration from a third reviewer (Jingwen Ruan). When needed, the authors of recent original studies were contacted to obtain additional information.

\section{Data extraction and management}

Both authors independently extracted the following data from each trial using EpiData 3.1 (ver. 270108, the EpiData Association): 1) basic article information: first author, year, language and country of publication, title; 2) inclusion and exclusion criteria; 3 ) study baseline: the number of study groups and center, sample size, sex ratio, age and course; 4) interventions in the observation group and the control group (type, frequency and duration); (5) outcome; 6) methodological quality, and hence, risk of bias.

\section{Assessment of risk of bias in included studies}

We used the Cochrane Handbook for Systematic Reviews of Interventions to evaluate the quality of the reviews. ${ }^{25}$ Seven domains were assessed for each study: 1) random sequence generation, 2) outcome allocation concealment, 3) blinding of participants and personnel, 4) blinding of assessment, 5) incomplete outcome data, 6) selective reporting bias and 7) other bias. Two authors (Liming Lu and Jingchun Zeng) independently assessed the studies by scoring each criterion as either "high risk", "low risk" or "unclear". If the results were inconsistent, they were resolved through reassment by a third reviewer (Guohua Lin).

\section{Measures of treatment effects}

For continuous measures, weighted mean differences were the preferred tool for analyzing results when outcome measures were identical. Standardized mean differences (SMDs) were used when different instruments had been used for the same outcome measurements.

\section{Unit of analysis issues}

We extracted data from the outcomes measured immediately after sessions had ended, for a period of up to 1 week.

\section{Assessment of heterogeneity}

When tests were similar, a heterogeneity test was performed on the included studies by using a Chi-test and $\mathrm{I}^{2}$ test to estimate the included studies. The $P$-value of the Chi-test was used to test the heterogeneity. When $P<0.1$, there was heterogeneity; when $P>0.1$, there was no obvious heterogeneity.

\section{Assessment of reporting bias}

We used the Cochrane Handbook for Systematic Reviews of Interventions to evaluate review quality. When results were inconsistent, evaluators cross-checked them. They were then resolved by reassessment from a third reviewer (Guohua Lin).

\section{Data synthesis}

When there was sufficient similarity in the data, we used RevMan5.1 for analysis. When there was insufficient similarity, we used descriptive analysis. The count data were represented with RR, while the continuous variables were represented with SMD. If $\mathrm{I}^{2}$ was $<50 \%$ and the P-value was $>0.1$, we used a fixed-effects model. If $\mathrm{I}^{2}$ was $>50 \%$ and the $P$-value was $<0.1$, this showed that statistical heterogeneity existed among the studies. When there was no clinical heterogeneity, a random-effects model was used. If the pooled results had clinical heterogeneity, 
subgroup analysis was performed based on patient characteristics and control interventions. The outcome measurement tools and sensitivity analysis were adapted to handle this, when necessary.

The GRADE approach was utilized to assess the overall quality of the evidence. This grading method for each outcome considered risk of bias, inconsistency of results, indirectness of evidence, imprecision and publication bias.

\section{Subgroup analysis and investigation of heterogeneity}

Subgroup analysis was conducted for individuals with different interventions (eg, different types of acupuncture and treatment durations) and different measurement tools.

\section{Results}

\section{Description of studies}

The initial search returned a total of 1,250 articles, including database queries and other sources. After removing the duplicates, we screened the abstracts of 876 articles. We read the full texts of 49 articles and excluded 827.8 articles were included in the final qualitative synthesis. The study flow is illustrated in Figure 1.

We ended up including 8 articles. Two studies were in the English language, and the rest were in Chinese. In these articles, acupuncture-related techniques such as electroacupuncture (EA), fire needle, acupoint injection and cupping were used to treat PHN. Drugs such as indomethacin, lofen, carbamazepine, and pregabalin were used in the control group. The study characteristics for each included trial are shown in Table 1.

\section{Risk of bias in included studies}

The recommendations in the methods section of the Cochrane Handbook 5.1.0 were used to assess the risk of bias. All included studies mentioned the randomization methods used. Six used a random number table to divide patients into experimental and observation groups. Two studies used a computer-generated random number.

In one study, sham EA was used to blind the patients. ${ }^{26}$ Seven studies mentioned blinding details for neither participants nor administrators. Because of the intervention
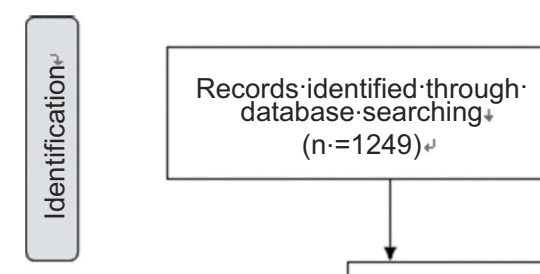
database-searching $(n \cdot=1249)$
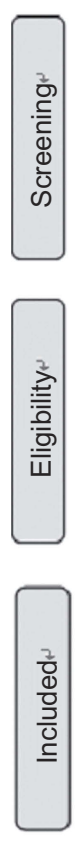

Assitional-records·identifies. through-other-sources $\downarrow$ $(n \cdot=1)$

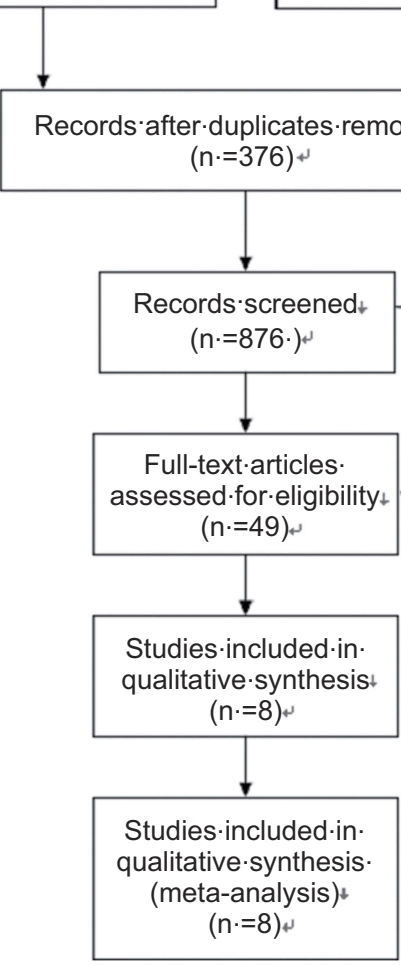

Figure I Flow diagram of study selection and identification. 


\begin{tabular}{|c|c|c|c|c|c|c|c|c|}
\hline 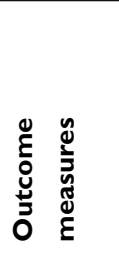 & 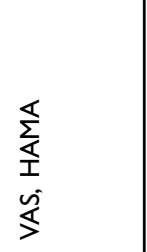 & 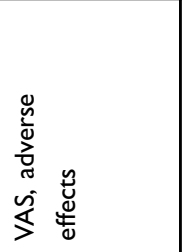 & $\underline{s}$ & 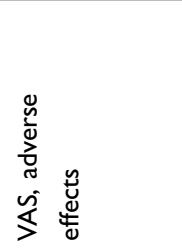 & 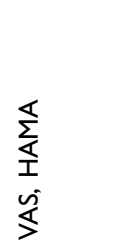 & $\begin{array}{l}\overrightarrow{0} \\
0 \\
v \\
\dot{y}\end{array}$ & 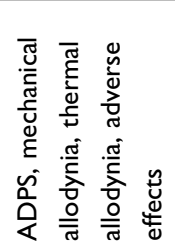 & 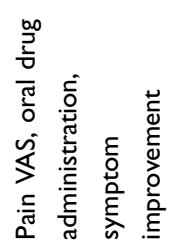 \\
\hline \multirow[b]{2}{*}{ 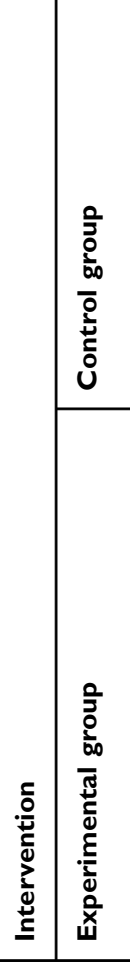 } & 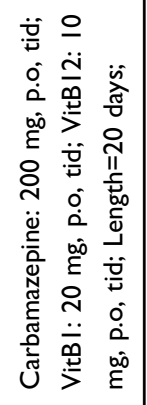 & 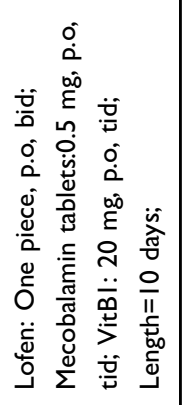 & 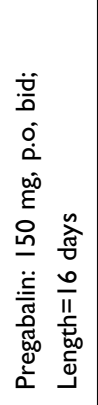 & 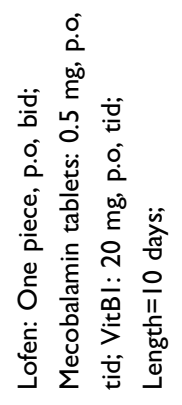 & 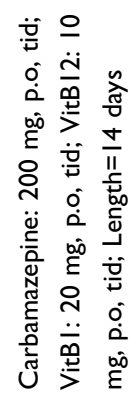 & 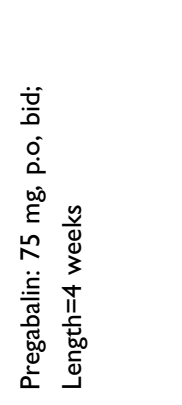 & 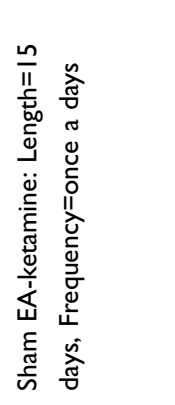 & 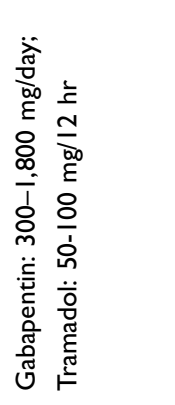 \\
\hline & 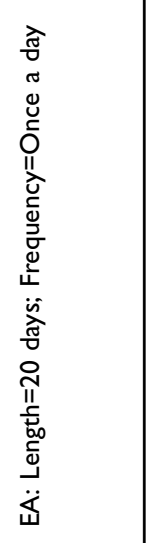 & 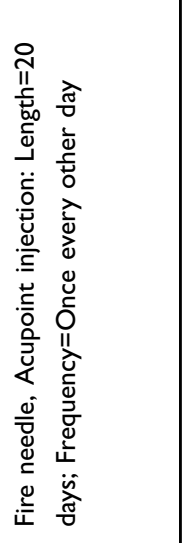 & 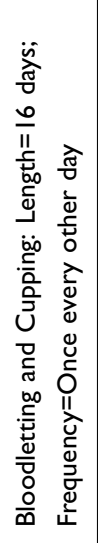 & 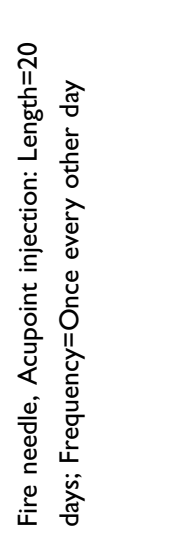 & 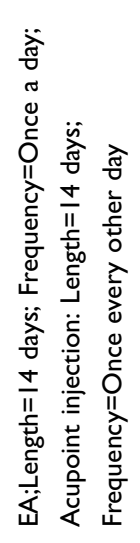 & 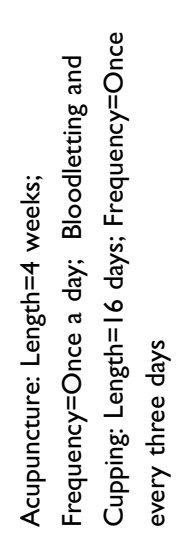 & 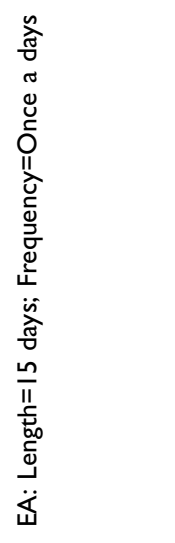 & 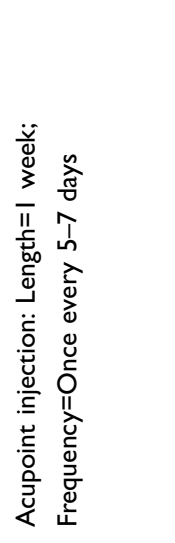 \\
\hline 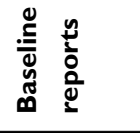 & 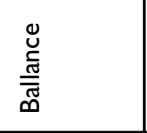 & 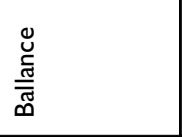 & 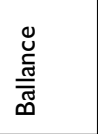 & 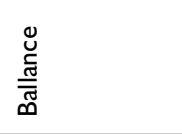 & 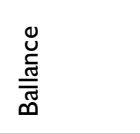 & 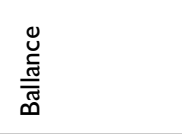 & 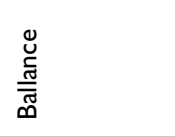 & 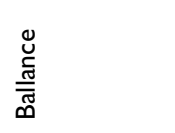 \\
\hline 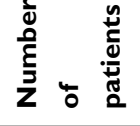 & 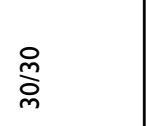 & $\frac{\text { ơ }}{\frac{+}{q}}$ & 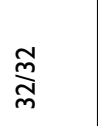 & $\frac{j}{f}$ & 商 & 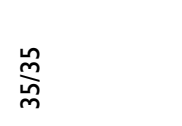 & $\stackrel{\bar{a}}{\underline{a}}$ & ্ㅗ \\
\hline 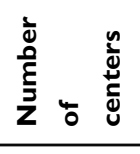 & - & - & $N$ & - & - & - & - & - \\
\hline 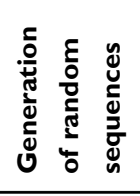 & 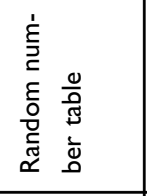 & 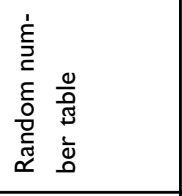 & 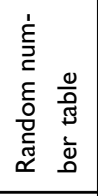 & 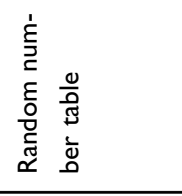 & 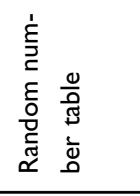 & & 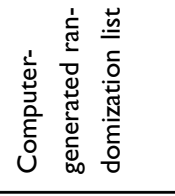 & 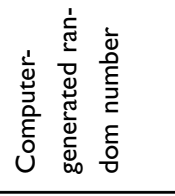 \\
\hline نे & 妾 & 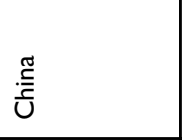 & 趇 & : & 胥 & 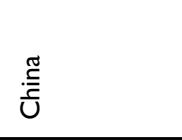 & $\stackrel{\widetilde{J}}{3}$ & 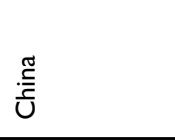 \\
\hline 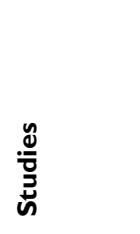 & $\begin{array}{l}\frac{0}{a} \\
\frac{1}{\alpha} \\
\overline{0} \\
\frac{0}{N}\end{array}$ & $\begin{array}{l}\frac{m}{2} \\
\stackrel{D}{N} \\
\Xi\end{array}$ & 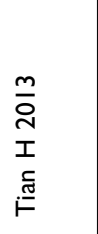 & 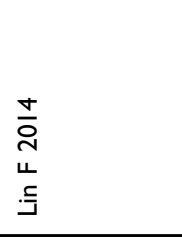 & 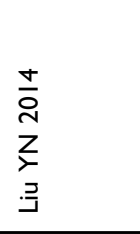 & 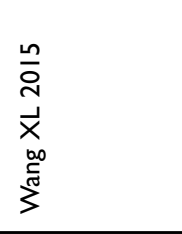 & 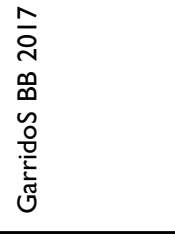 & $\begin{array}{l}\frac{\infty}{0} \\
\vdots \\
\square\end{array}$ \\
\hline
\end{tabular}


differences between the experimental group and the control group, it was difficult to established blindness for the whole research process. We contacted the authors, and the authors of two of the studies said that they had blinded both evaluators and statisticians. ${ }^{27,28}$ Another study said they had blinded the outcome assessments only. ${ }^{29}$ The authors of an additional study replied that they had not used blinding because it would have been easy to break. ${ }^{30}$ The authors of three of the studies did not reply to our inquiries.

All studies reported all outcomes which had been described by their respective methods sections, and no missing data were mentioned. Information was insufficient to analyze other potential sources of bias. The detailed risk of bias is shown in Figure 2. It assesses the quality of the included studies.

\section{Effects of interventions Outcome}

Primary outcome (pain intensity). Pain intensity was measured by VAS and ADPS. No study reported onset of pain relief time. Because different methods of acupuncture were used in the studies, we used SMD and 95\% CI to measure the effect of acupuncture on pain intensity. When there was statistically significant heterogeneity of results $\left(P<0.00001, \mathrm{I}^{2}=86 \%\right)$, a random-effects model was used. After intervention, the scores were lower in the acupuncture group than in the control group (SMD: $-1.78,95 \% \mathrm{CI}$ : -2.36 to $-1.21, P<0.00001$ ) (Figure 3 ).

Subgroup analysis. Secondary outcomes.

Different types of acupuncture. We conducted subgroup analysis based on the different types of acupuncture to assess whether the variation could have influenced the results. The results showed that acupuncture can relieve pain intensity; however, there are many different types of acupuncture (Figure 3).

Different sample sizes. We conducted subgroup analysis based on the varying sample sizes. In the acupuncture group, pain intensity decreased in studies with $<60$ samples (SMD: -0.48 , 95\%CI: -0.95 to -0.02 ). For studies with $\geq 60$ samples, acupuncture mitigated pain intensity (SMD:-2.19, 95\%CI: -2.43 to -1.95 ) (Figure 3).

In subgroup analysis based on variations in sample size, the statistical heterogeneity disappeared when we excluded two of the studies, ${ }^{26,27}$ Pain intensity in the acupuncture group was still lower (SMD: -2.19 , 95\% CI: -2.43 to $-1.95)$. Analysis showed that the results were stable and that the sample size could affect statistical heterogeneity.

Secondary outcomes.

Onset of pain relief time. Not reported in any studies.

Hamilton Anxiety Scale (HAMA). Two studies used the HAMA to evaluate the severity of anxiety symptoms in

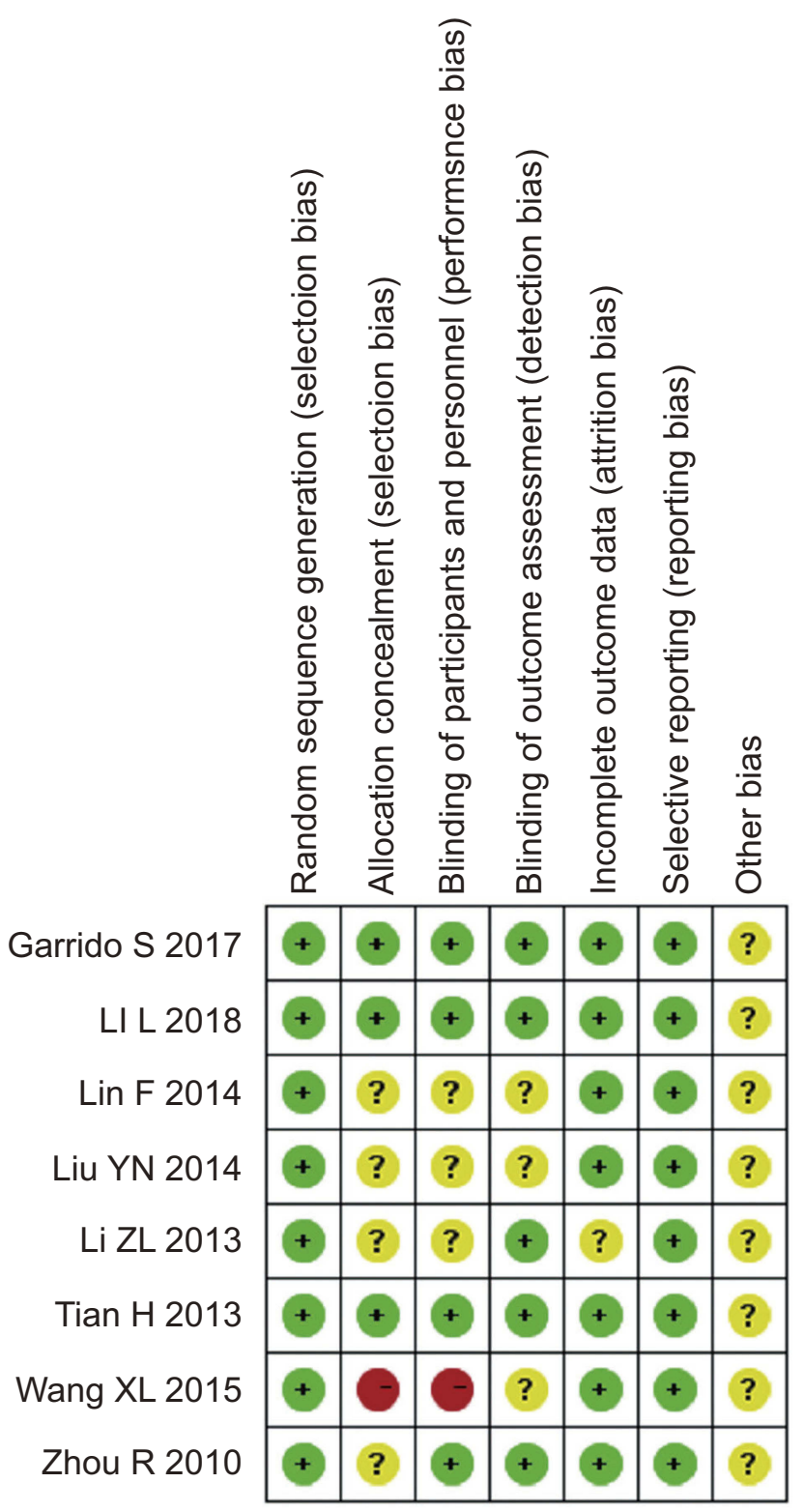

Figure 2 Risk of bias graph for included studies.

patients. Acupuncture lowered the HAMA score (SMD: $-18.94,95 \%$ CI: -37.37 to -0.52$)$ with high degrees of heterogeneity $\left(\mathrm{I}^{2}=98 \%, P<0.00001\right)$ (Figure 4$)$.

$Q O L$. QOL was reported in one study; the QOL was higher in the acupuncture group than in the control group (SMD: 3.78, 95\% CI: 2.50-5.06). Heterogeneity was not applicable because the number of studies included was insufficient (Figure 4).

Adverse effects. In the eight studies included, two mention adverse effects (Figure 4). One study reported side effects (nausea, vomiting, constipation and sedation) in eight patients from the sham group. ${ }^{26}$ In another study, adverse effects occurred in neither the acupuncture group nor the control group. 


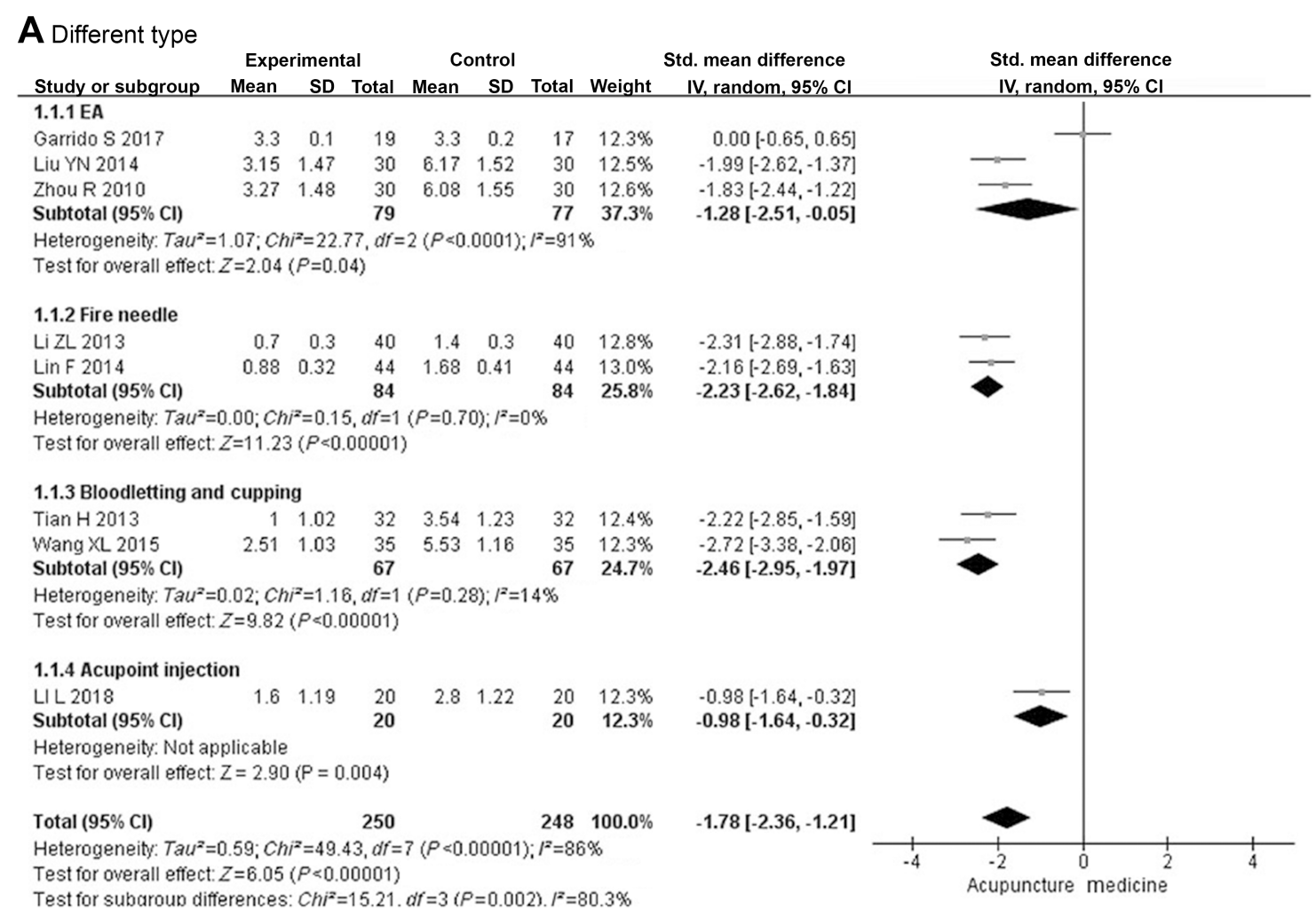

B Different sample size

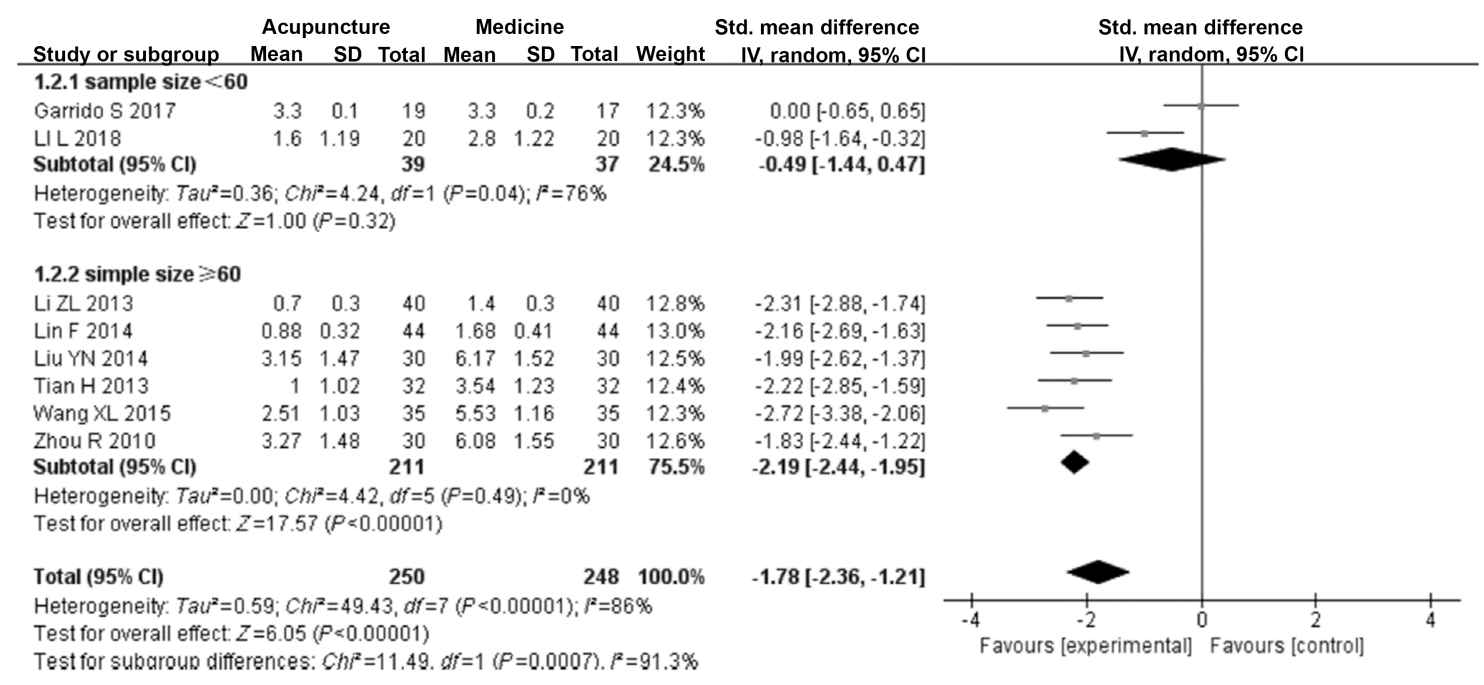

Figure 3 Forest plot of acupuncture for PHN. Outcome:pain intensity-VAS. (A) Subgroup: Based on different types of acupuncture therapy; (B) Subgroup: Based on different sample sizes.

Abbreviations: EA, electroacupuncture; PHN, postherpetic neuralgia; VAS, visual analogue scale.

\section{Publication bias}

A funnel plot of the included studies was created to evaluate publication bias (Figure 5). However, the number of included studies was small, and the sample sizes were insufficient. Thus, it was difficult to assess publication bias by funnel plot. Therefore, publication bias could have been misleading, and thus was not performed in the review. ${ }^{31}$

\section{Quality levels of evidence}

The Cochrane Collaboration Network GRADE was used to assess the quality of evidence for the systematic analysis. We assessed acupuncture for PHN of pain intensity, onset of pain relief time, HAMA, QOL and adverse effects. Details are shown in the GRADE evidence profile and the summary of findings table. The results show that the quality of evidence 
A Hamilton anxiey scale(HAMA)

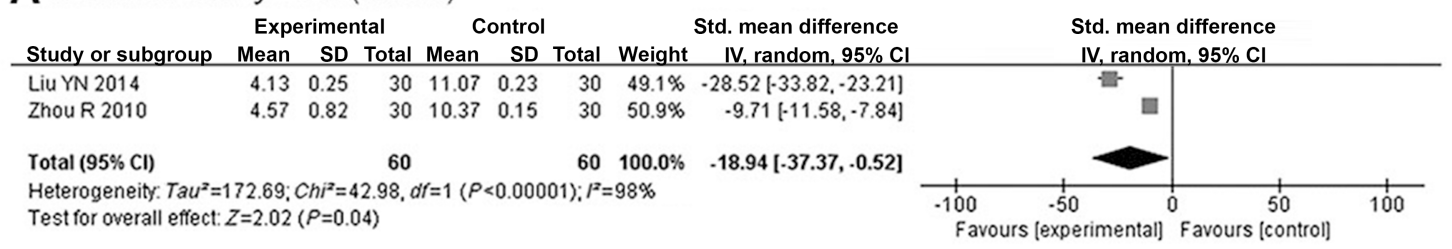

B Quality of life(QOL)

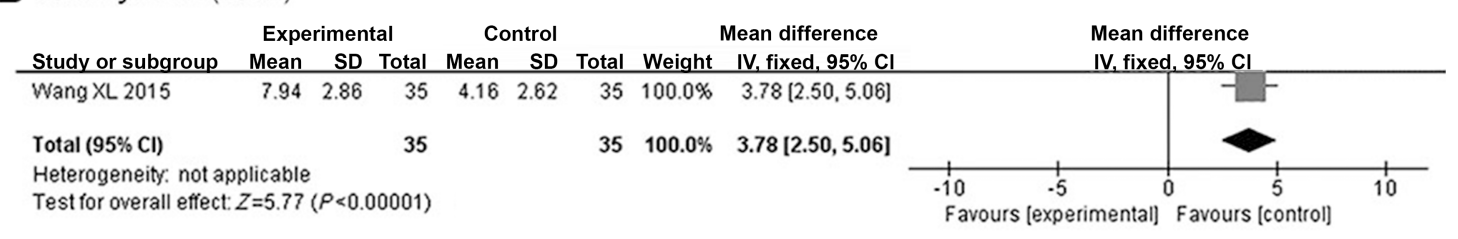

C Adverse effects

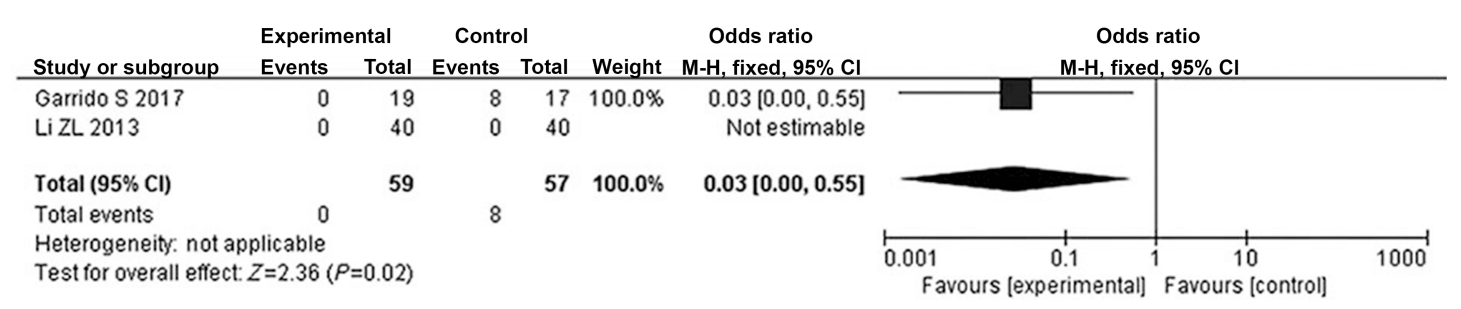

Figure 4 Forest plot of acupuncture for PHN. Outcome: pain intensity-VAS. Subgroup: Based on different sample sizes.

Abbreviations: EA, electroacupuncture; $\mathrm{PHN}$, postherpetic neuralgia.

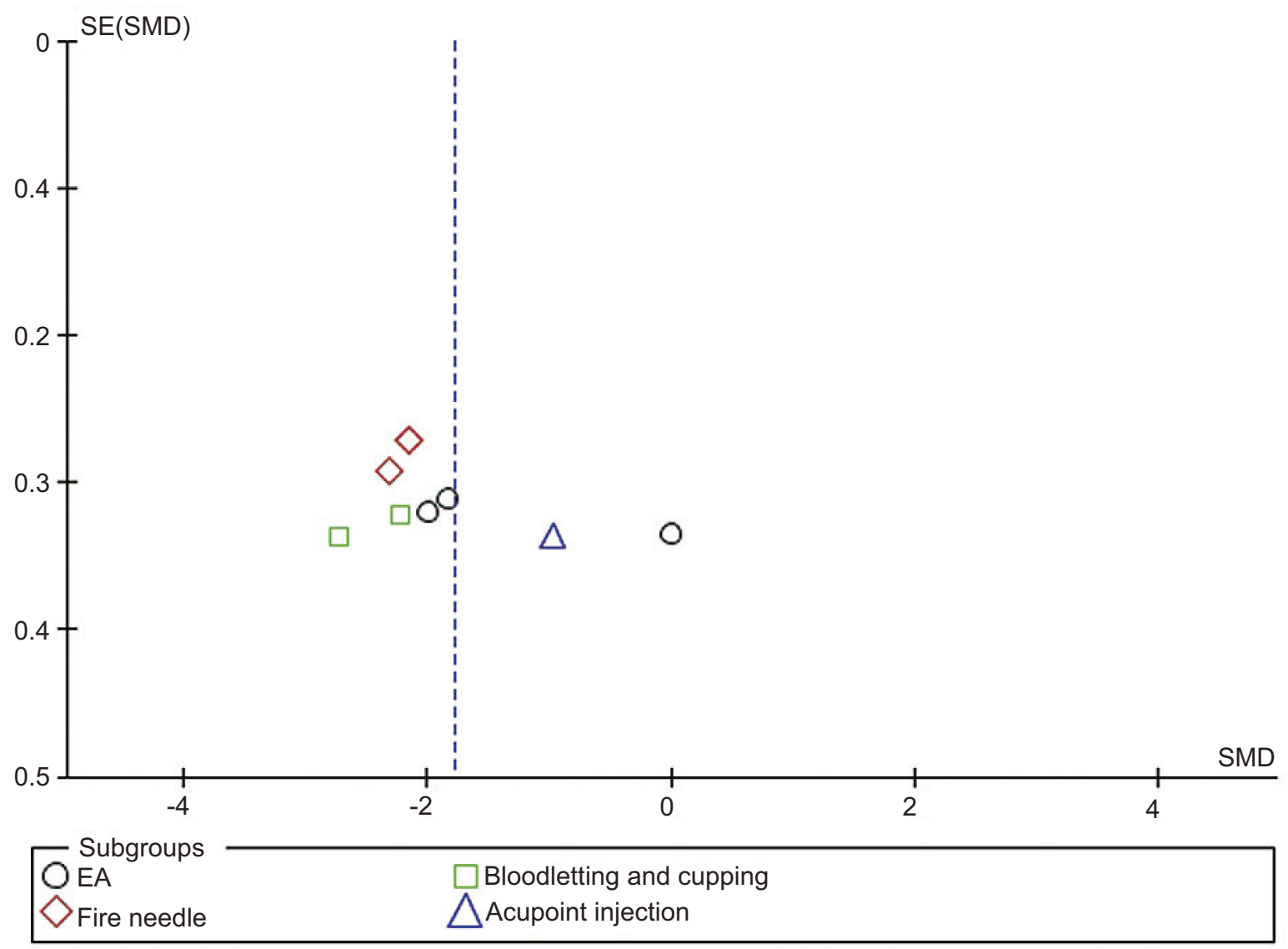

Figure 5 Funnel plot of publication bias of acupuncture for PHN. Outcome: pain intensity. Subgroup: different methods. Abbreviation: $\mathrm{PHN}$, postherpetic neuralgia. 
was moderate for the assessment of pain intensity. Because the experimental groups and control groups used different methods, it was difficult to apply blinding to the patients. Therefore, we did not downgrade the quality of evidence for risk of bias. However, the number of included studies was insufficient to assess publication bias, and thus it was downgraded. At the onset of pain relief time, the quality of evidence was low because of the lack of blinding and the small sample sizes of the included studies. For HAMA, the quality of evidence was low due to the low $P$-value and the large $\mathrm{I}^{2}$. In both QOL and adverse effects, the quality of evidence was low for the small number of studies and for the small population size. A detailed summary of findings table and GRADE evidence profile are provided in the Supporting Information section (S3 summary of findings table and GRADE evidence profile).

\section{Discussion}

\section{Summary of main results}

This systematic review includes eight prospective studies containing 498 patients. The meta-analysis showed that pain, the primary outcome, was lower in the acupuncture group than in the control group. Subgroup analysis of the different types showed that EA, fire needling, bloodletting and cupping each relieved more pain than the controls. When the sample size was greater than 60 , the acupuncture group showed better relief (ie, VAS score) than the control group. However, there was no significance for sample sizes less than 60. Subgroup analysis showed that the sample size could have affected statistical heterogeneity.

Based on these results, less literature was included in the secondary outcome. In the HAMA outcome, acupuncture was shown to relieve anxiety. These results were significant with a high $\mathrm{I}^{2}$ and a low $P$-value. Only one study was included for QOL, and in it, the acupuncture group had better QOL than the control group. ${ }^{30}$ No adverse reactions occurred in the acupuncture groups of the two studies that reported on them.

\section{Interpreting the findings}

It is believed that acute zoster infection in nerve damage results in persistent pain which is the main symptom of PHN. ${ }^{32}$ Depression and social communication disorder are also associated with pain in postherpetic neuralgia patients. $^{33}$ The European Federation of Neurological Societies (EFNS) recommends tricyclic antidepressants (TCAs) and gabapentin/pregabalin as first-line treatments for PHN. ${ }^{34}$ However, their side effects must be taken into consideration, particularly with elderly patients. ${ }^{14}$ China's guidelines for treatment of PHN recommend acupuncture because of its clinical effects. ${ }^{35}$ A search of the clinical trial registries showed that large-sample randomized controlled trials of acupuncture for PHN are lacking. Additionally, no rigorous systematic reviews were found either.

This review includes studies of acupuncture treatment for PHN. The meta-analysis shows that acupuncture can reduce pain intensity, relieve anxiety and improve QOL among PHN patients. In the acupuncture group, the incidence of adverse effects was lower than in the control group. These results are similar to those of existing published studies. The VZV Pain Study Group reported that acupuncture could play a role in relieving acute herpetic pain, and no serious treatment-related adverse events were observed in the study. ${ }^{36}$

\section{Limitations}

This review has several limitations. Although we searched the Cochrane Central Register of Controlled Trials (CENTRAL), MEDLINE, EMBASE and WHO International Clinical Trials Registry Platform, we found few English language studies of acupuncture for PHN. Furthermore, there have been persistent discrepancies in the definition of PHN. Some have reported that pain persisting 30 days or more after rash onset can be defined as PHN, while others have used persistent pain for 120 days as their definition. Although similar reviews have published evidence for the efficacy and safety of acupuncture for PHN, we sought more information, focusing on the advantages of acupuncture therapy over pharmacologic therapy. ${ }^{37}$ Through our review, there is a need for more studies to conduct subgroup analyses on pain persistence.

\section{Clinical implications}

This review finds that acupuncture is beneficial for PHN, especially for associated pain relief. It can provide a treatment option for clinicians worried about adverse effects of drugs, and evidence-based evidence for acupuncturist. The findings of this review support the view that acupuncture is an effective PHN treatment.

\section{Conclusion}

This study has shown that acupuncture may reduce pain intensity, relieve anxiety and improve the QOL of PHN patients. No adverse effects were reported for treatment with acupuncture. Different types of acupuncture such as 
EA, fire needle, bloodletting and cupping can reduce pain intensity. Sample size may also affect the results of studies.

The GRADE Working Group grades for the evidence of acupuncture's effect on PHN pain intensity were of moderate quality. For HAMA, QOL and adverse reactions, the quality of the evidence was low. Therefore, multicenter RCTs with large sample sizes and high methodological quality are needed. This will lead to a deeper understanding of acupuncture for PHN.

\section{Acknowledgments}

This research was supported by the National Natural Science Foundation of China (grant no. 81574061), the First-class Discipline Construction Foundation of Guangzhou University of Chinese Medicine (Chinese medicine department), the Young Top Talent Project of Scientific and Technological Innovation in Special Support Plan for Training High-level Talents in Guangdong (grant no. 2017TQ04R627) and the Guangdong Natural Science Foundation (grant no. 2016A030310290).

\section{Author contributions}

All authors contributed to data analysis, drafting or revising the article, gave final approval of the version to be published, and agree to be accountable for all aspects of the work.

\section{Disclosure}

The authors report no conflicts of interest in this work.

\section{References}

1. Dworkin RH, Schmader KE. Treatment and prevention of postherpetic neuralgia. Clin Infect Dis. 2003;36(7):877-882. [published Online First: 2003/03/26]. doi:10.1086/368196

2. Feller L, Khammissa RAG, Fourie J, et al. Postherpetic neuralgia and trigeminal neuralgia. Pain Res Treat. 2017;2017:1681765.

3. Watson CP, Oaklander AL. Postherpetic neuralgia. Pain Pract. 2002;2 (4):295-307. [published Online First: 2006/12/13]. doi:10.1046/ j.1533-2500.2002.02039.x

4. Dworkin RH, Portenoy RK. Pain and its persistence in herpes zoster. Pain. 1996;67(2-3):241-251. [published Online First: 1996/10/01].

5. Hope-Simpson RE. The nature of herpes zoster: a long-term study and a new hypothesis. Proc R Soc Med. 1965;58:9-20. [published Online First: 1965/01/01].

6. Johnson RW. The future of predictors, prevention, and therapy in postherpetic neuralgia. Neurology. 1995;45(12 Suppl 8):S70-S2. [published Online First: 1995/12/01]. doi:10.1212/wnl.45.12_suppl_8.s70

7. Petersen KL, Rowbotham MC. Natural history of sensory function after herpes zoster. Pain. 2010;150(1):83-92. [published Online First: 2010/05/11]. doi:10.1016/j.pain.2010.04.005

8. Dworkin RH, Gnann JW Jr., Oaklander AL, Raja SN, Schmader KE, Whitley RJ. Diagnosis and assessment of pain associated with herpes zoster and postherpetic neuralgia. J Pain. 2008;9(1 Suppl 1):S37-S44. [published Online First: 2008/01/31]. doi:10.1016/j.jpain.2007.10.008
9. Forbes HJ, Thomas SL, Smeeth L, et al. A systematic review and meta-analysis of risk factors for postherpetic neuralgia. [published Online First: 2015/07/29]. Pain. 2016;157(1):30-54. doi:10.1097/j. pain.0000000000000307

10. Forbes HJ, Bhaskaran K, Thomas SL, et al. Quantification of risk factors for postherpetic neuralgia in herpes zoster patients: a cohort study. Neurology. 2016;87(1):94-102. doi:10.1212/WNL.0000000000002808

11. Dubinsky RM, Kabbani H, El-Chami Z, Boutwell C, Ali H. Practice parameter: treatment of postherpetic neuralgia: an evidence-based report of the Quality Standards Subcommittee of the American Academy Of Neurology. Neurology. 2004;63(6):959-965. [published Online First: 2004/09/29]. doi:10.1212/01.wnl.0000140708.62856.72

12. Massengill JS, Kittredge JL. Practical considerations in the pharmacological treatment of postherpetic neuralgia for the primary care provider. J Pain Res. 2014;7:125-132. doi:10.2147/JPR.S57242

13. Harden RN, Kaye AD, Kintanar T, Argoff CE. Evidence-based guidance for the management of postherpetic neuralgia in primary care. Postgrad Med. 2013;125(4):191-202. [published Online First: 2013/ 08/13]. doi:10.3810/pgm.2013.07.2690

14. Finnerup NB, Attal N, Haroutounian S, et al. Pharmacotherapy for neuropathic pain in adults: a systematic review and meta-analysis. [published Online First: 2015/01/13]. Lancet Neurol. 2015;14 (2):162-173. doi:10.1016/s1474-4422(14)70251-0

15. Whitley RJ, Volpi A, Mckendrick M, Wijck AV, Oaklander AL. Management of herpes zoster and post-herpetic neuralgia now and in the future. J Clin Virol. 2010;48(Suppl 1):S20-S28. doi:10.1016/S1386-6532 (10)70005-6

16. Yeung WF, Chung KF, Tso KC, Zhang S-P, Zhang Z-J, Ho L-M. Electroacupuncture for residual insomnia associated with major depressive disorder: a randomized controlled trial. Sleep. 2011;34 (6):807-815. doi:10.5665/SLEEP.1056

17. Essex H, Parrott S, Atkin K, et al. An economic evaluation of Alexander Technique lessons or acupuncture sessions for patients with chronic neck pain: a randomized trial (ATLAS). PLoS One. 2017;12(12):e0178918. doi:10.1371/journal.pone.0178918

18. Davis RT, Badger G, Valentine K, Cavert A, Coeytaux RR. Acupuncture for chronic pain in the vermont medicaid population: a prospective, pragmatic intervention trial. Global Adv Health Med. 2018;7:2164956118769557. doi:10.1177/2164956118769557

19. MacPherson H, Vertosick EA, Foster NE, et al. The persistence of the effects of acupuncture after a course of treatment: a meta-analysis of patients with chronic pain. Pain. 2017;158(5):784-793. doi:10.1097/ j.pain.0000000000000747

20. Seo B-K, Han K, Kwon O, Jo D-J, Lee J-H. Efficacy of bee venom acupuncture for chronic low back pain: a randomized, doubleblinded, sham-controlled trial. Toxins. 2017;9(11):361. doi:10.3390/ toxins 9110361

21. Koh W, Kang K, Lee YJ, et al. Impact of acupuncture treatment on the lumbar surgery rate for low back pain in Korea: a nationwide matched retrospective cohort study. PLoS One. 2018;13(6):e199042. doi:10.1371/journal.pone. 0199042

22. Takahashi H. Effects of scalp acupuncture and auricular therapy on acute herpetic pain and postherpetic neuralgia: a case series. Med Acupuncture. 2007;19(2):113-120. doi:10.1089/acu.2007.0521

23. Li W, Peng W, Zhou J, Liu Z. Acupuncture for postherpetic neuralgia: a systematic review protocol. BMJ Open. 2014;4(11):e005725e25. doi:10.1136/bmjopen-2014-005725

24. Kotlyar A. Treatment of facial pain with I ching balance acupuncture. Med Acupuncture. 2017;29(6):405-410. doi:10.1089/acu.2017.1251

25. Higgins JP, Altman DG, Gotzsche PC, et al. The Cochrane Collaboration's tool for assessing risk of bias in randomised trials. BMJ. 2011;343:d5928. [published Online First: 2011/10/20]. doi:10.1136/bmj.d5928

26. Garrido-Suárez BB, Garrido G, Menéndex AB, et al. Combination of low frequency electroacupuncture plus subdissociative doses of ketamine in post-herpetic neuralgia patients. A pilot study. $J$ Pharm Pharmacogn Res. 2017;5(6):381-393. 
27. Li L, Shen L, Wang H, Lu Z. Jiaji acupoint injection of salvia miltiorrhiza-extract combined with SNB affect PHN. Int J Clin Exp Med. 2018;11(2):1141-1149.

28. Tian H, Tian YJ, Wang B, et al. Impacts of bleeding and cupping therapy on serum $\mathrm{P}$ substance in patients of postherpetic neuralgia. Zhongguo Zhen Jiu. 2013;33(8):678.

29. Zou R, Hong-Xing Z, Yun XU. Clinical study of electroacupuncture in paravertebral points combined with surrounding acupuncture on postherpetic neuralgia. J Emergency Traditional Chin Med. 2010;19 (7):1109-1110

30. Wang XLZF, Xu AX. Clinical observation on treatment of postherpetic neuralgia with Elongated Needle Combined with cupping and cupping therapy. J Parct Chin Med. 2015;31(10):943-944.

31. Lau J, Ioannidis JPA, Terrin N, Schmid CH, Olkin I. The case of the misleading funnel plot. BMJ. 2006;333(7568):597-600. doi:10.1136/ bmj.333.7568.597

32. Johnson RW, Rice AS. Clinical practice. Postherpetic neuralgia. $N$ Engl J Med. 2014;371(16):1526-1533. [published Online First: 2014/ 10/16]. doi:10.1056/NEJMcp1403062
33. Staats PS. Pain, depression and survival. Am Fam Physician. 1999;60 (1):42,44. [published Online First: 1999/07/22].

34. Attal N, Cruccu G, Baron R, et al. EFNS guidelines on the pharmacological treatment of neuropathic pain: 2010 revision. [published Online First: 2010/04/21]. Eur J Neurol. 2010;17(9):e1113-e88. doi:10.1111/j.1468-1331.2010.02999.x

35. Neuralgia Egftdatop. Chinese experts consensus on diagnosis and treatment of postherpetic neuralgia. Chin J Pain Med. 2016;22 (3):161-167.

36. Ursini T, Tontodonati M, Manzoli L, et al. Acupuncture for the treatment of severe acute pain in herpes zoster: results of a nested, open-label, randomized trial in the VZV pain study. $B M C$ Complement Altern Med. 2011;11:46. [published Online First: 2011/ 06/07]. doi:10.1186/1472-6882-11-46

37. Wang Y, Li W, Peng W, Zhou J, Liu Z. Acupuncture for postherpetic neuralgia: systematic review and meta-analysis. Medicine. 2018;97 (34):e11986-e86. doi:10.1097/MD.0000000000011986

\section{Publish your work in this journal}

The Journal of Pain Research is an international, peer reviewed, open access, online journal that welcomes laboratory and clinical findings in the fields of pain research and the prevention and management of pain. Original research, reviews, symposium reports, hypothesis formation and commentaries are all considered for publication. The manuscript management system is completely online and includes a very quick and fair peer-review system, which is all easy to use. Visit http:// www.dovepress.com/testimonials.php to read real quotes from published authors. 